\title{
Twin roller quenching technique and preparation of glasses of high melting ionic solids
}

\author{
K B R VARMA \\ Materials Research Laboratory. Indian Institute of Science. Bangalore 560012, Inda \\ MS recoved 2 February 1987
}

\begin{abstract}
A rapid quenching technque with a quenching rate of roughly $10^{6} \mathrm{C} / \mathrm{sec}$ has been developed to prepare glassy simples of $\mathrm{ABO}_{3}$ type materials. Glasses of potassium lathium niobate have heen prepared by this technique. These glasses have been characterized by $x$-ray diffaction. electron diffaction and differential scanning calorimetry techniques to wisess the quality of the obtained glasses.
\end{abstract}

Keywords. Twin roller quenching technique; potassium lithium niobate glass.

\section{Introduction}

Ferroelectric-like dielectrc anomaltes 'lave been reported in glassy phases of a few $\mathrm{ABO}_{3}$ materials (Glass et al 1977). Several technological applications are possible for such materials and it is therefore natural to investigate whether this curious phenomenon is universal to the amorphous phases of the large class of $\mathrm{ABO}_{3}$-type compounds. It is now widely known that glassy phases of $\mathrm{ABO}_{3}$ materials can be obtained by several techniques, notably by fast quenching of melts using twin roller and other techniques and r.f. sputtering. While sputtering techniques are useful, it is rather difficult to obtain from this technique thick films suitable for a number of physicochemical studies. Further. non-stoichiometry of sputtered samples also creates difficulties in understanding their behaviour. Fast quenching techniques are thus quite advantageous for sample preparation. They provide high quenching rates $\left.1 \sim 10^{6} \mathrm{C} \mathrm{sec}{ }^{1}\right)$ and are relatively easy to fabricate. Thick and uniform flakes of glasses of many non-metallic materials can be readily prepared.

In this communication, fabrication of a twin roller quenching apparatus is described. Preparation of flaky glass samples of potassium lithium niobate (KLN) is described along with a preliminary report of the structural and thermal properties of the KLN glass.

\section{Twin roller set-up}

The twin roller quenching apparatus consists of two hard chrome-plated copper rollers whose dimensions were $5 \mathrm{~cm}$ (dia) $\times 10 \mathrm{~cm}$ (length) held together in a heavy steel frame (figure 1). One of the rollers is associated with a cone pulley arrangement and is driven by an a.c. motor with a single belt-drive to synchronize the rotation speeds of both rollers upto 3000 r.p.m. The rotation speeds of the rollers would be varied by suitably positioning them on the cone pulley arrangement. In this set-up, 

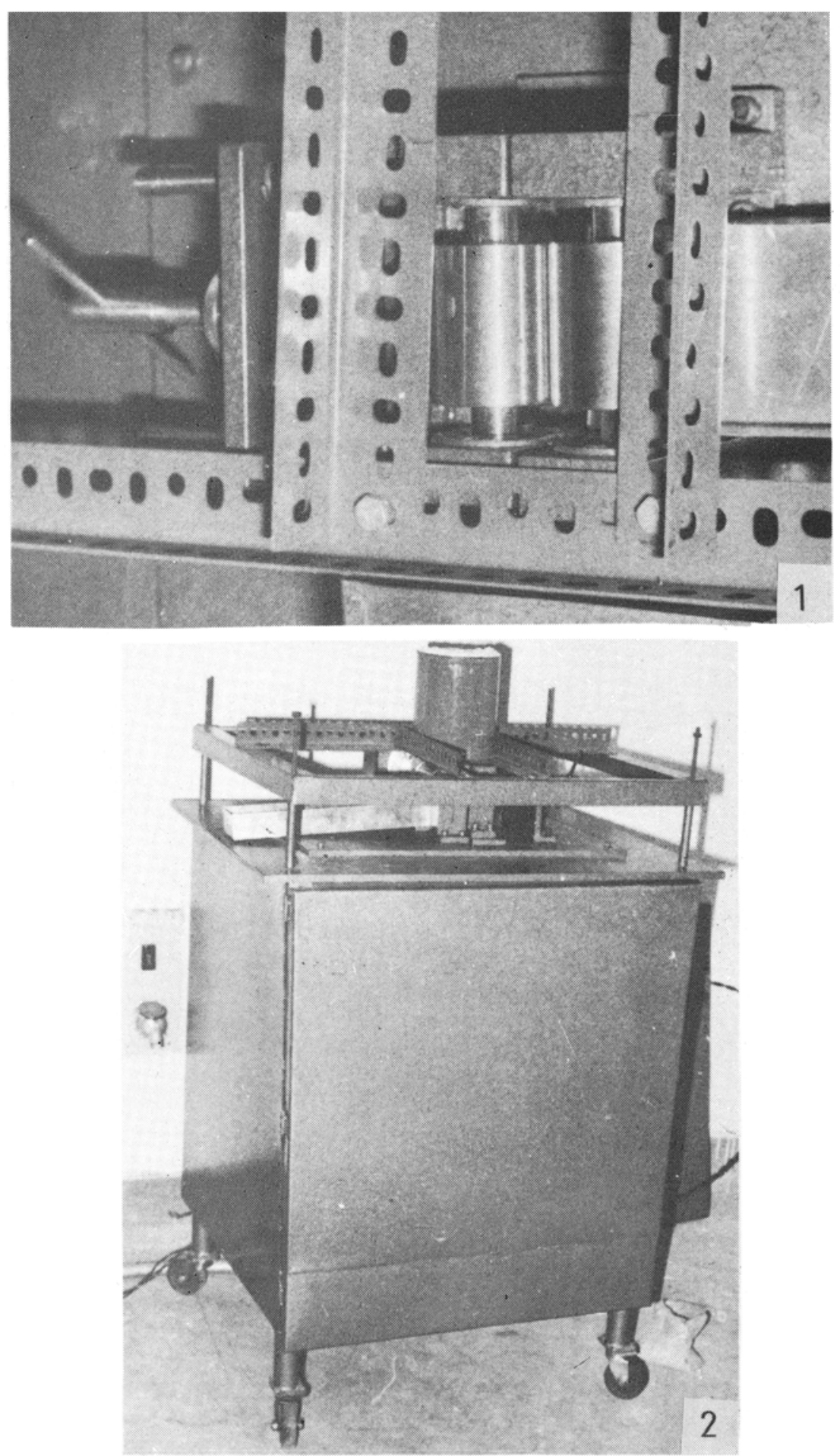

Figures 1-2 1. Top view of the twin roller assembly. 2. The whole arrangement of twin roller set-up. 
three speeds, ie. 1500,2000 and 3000 r.p.m. could be chosen. One of the rollers is mounted with a fixed central axis of rotation whereas the second one is springloaded and can be horizontally translated towards the fixed roller. This enables gap adjustment between the rollers, a feature necessary for fixing the flake thickness. This is an additional advantage of the present design. The spring system shown in figure 1 also helps in maintaining a uniform gap between the rollers during the operation of the instrument. The total set-up is shown in figure 2. It has been found possible to obtain moderately large size flakes of uniform thickness by a careful manipulation of the above parameters. Only when the molten samples wet the surface, longer ribbons of the quenched material can be obtained. The heating assembly shown in figure 2 consists of a quartz crucible with $1 \mathrm{~mm}$ (dia) hole. The inner surface of the crucible is wrapped with a platinum foil (which prevents contamination of the samples). The crucible is surrounded by a wire wound furnace. It is however possible to replace the furnace by a ring type gas burner, particularly while melting oxides of very high melting points. The furnace assembly is itself mounted on a sliding platform (figure 2) which is positioned on top of the roller assembly. The centering of the melt flow from the crucible is adjusted through the movement of the furnace itself along with the crucible. The present design is a modified version of a quenching set-up described by Chen and Miller (1970).

\section{Potassium lithium niobate glass}

Crystalline $\mathrm{KLN}\left(\mathrm{K}_{3} \mathrm{Li}_{2} \mathrm{Nb}_{5} \mathrm{O}_{15}\right)$ required for glass preparation was prepared starting from $\mathrm{K}_{2} \mathrm{CO}_{3}, \mathrm{Li}_{2} \mathrm{CO}_{3}$ and $\mathrm{Nb}_{2} \mathrm{O}_{5}$. A mixture containing alkali carbonates and $\mathrm{Nb}_{2} \mathrm{O}_{5}$ in required proportions was ground together and mixed well. It was initially heated in a platinum crucible for about $8 \mathrm{hr}$. The product was cooled and again ground to a fine powder. The powder was compacted and heated slowly to $\sim 1250 \mathrm{~K}$. It was heated at this temperature for about $48 \mathrm{hr}$ and slowly cooled to room temperature. The $x$-ray diffraction of the calcined product was examined and the formation of KLN was confirmed.

In order to make the glass, about $100 \mathrm{~g}$ of the powder was melted in the crucible described earlier. The temperature of the melt was raised by about $80-100 \mathrm{~K}$ above the melting temperature $(1280 \mathrm{~K})$. The melt was gently coaxed out through the capillary either by shaking or by directing a hot air blower perpendicularly over the surface of the melt in the crucible. The gentle pressure on the melt can be kept in order to maintain a continuous jet of the flowing melt. The molten jet is allowed to take the space between the rollers already set in rotation. The free fall distance of the melt before reaching the rollers is about $1 \mathrm{~cm}$. The quenched flakes were collected in a tray kept just below the roller assembly.

Flakes obtained from quenching $K L N$ werc quite transparent. Their thickness could be varied from $50100 \mu \mathrm{m}$. The quenching rates were estimated by the procedure used by Chen and Miller (1970) and were found to be $\sim 10^{5} 10^{6} \mathrm{C} / \mathrm{sec}$. The flakes were examined using $x$-ray diffraction, high resolution electron microscopy and differential scanning calorimetry (DSC). The glassy nature of the sample is quite obvious from the broad peaks in $\mathrm{x}$-ray diffraction (figure 3) and from the appearance of two broad haloes in the electron diffraction (inset in figure 4). The high resolution image shows presence of fine, short range fringes which criss-cross the entire image 


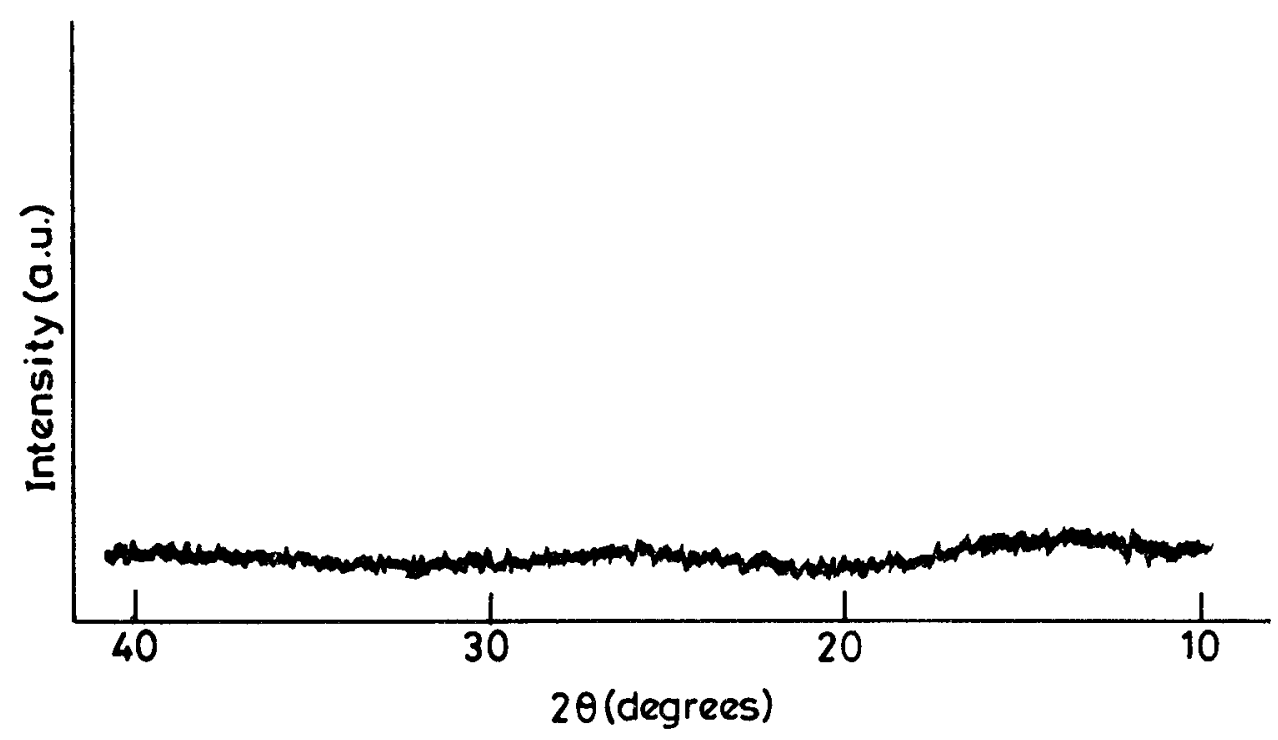

Figure 3. X-ray diffraction pattern of the quenched flake of KLN.

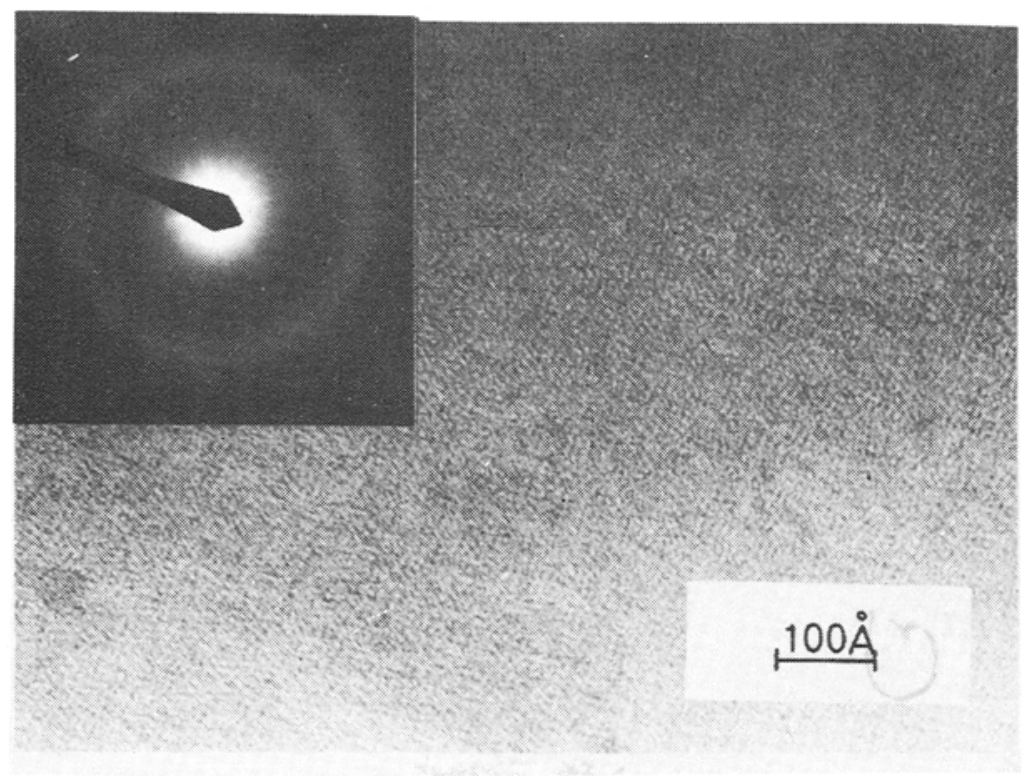

Figure 4. Electron diffraction pattern of the quenched flake of KLN.

field. Such ultra microstructures have been noted both in sputtered films (Varma et al 1985) and in some glassy metals.

DSC scan of the glassy sample is shown in figure 5. Glass transition occurs at $570 \mathrm{~K}$ and the sample crystallizes around $825 \mathrm{~K}$. Crystallized samples are opaque. Unfortunately, we could not show the crystallization temperature in the above figure. However, the glassy flakes have been heated at various temperatures followed by electron microscopic studies to determine the crystallization temperature. 


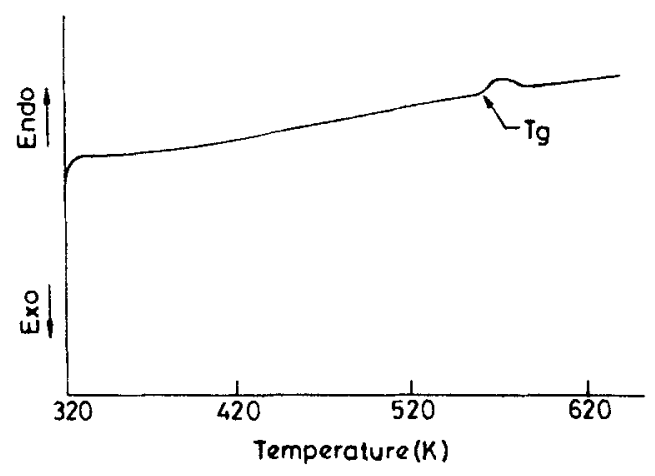

Figure 5. Typical DSC trace for as quenched flake of $\mathrm{KLN}$ (heating rate $20 \mathrm{~K} / \mathrm{m} / \mathrm{n}$ ).

Further investigations are underway to determine whether the regions producing fringes are truly microcrystalline clusters and whether they correspond to the dielectrically soft units described by Lines (1977) and which give rise to dielectric anomalies. Since $\mathrm{LiNbO}_{3}$ exhibits dielectric anomalies both in r.f. sputtered films and in bulk glass form, we expect similar dielectric behaviour even in mixed alkali niobates.

In conclusion, this note describes a simple rapid quenching apparatus built for obtaining glassy phases of high melting ionic solids and preparation and preliminary characterization of a typical mixed alkali niobate glass.

\section{Acknowledgements}

The author is indebted to Prof. K J Rao for helpful suggestions and critical reading of the manuscript and to Prof. C N R Rao for kind and continued encouragement.

\section{References}

Chen H S and Miller C E 1970 Ret. Sct. Instr. 411237

Glass A M, Lines M E. Nassau K and Shiever J W 1977 Appl. Phy's. Lett. 31249

Lines M E 1977 Phy's. Ret. B15 388

Varma K B R. Harshavardhan K S, Rao K J and Rao C N R 1985 Mater. Res. Bull. 20315 